\title{
Eliminating nonlinear phase mismatch in resonantly enhanced 4-wave mixing
}

\author{
Mattias Johnsson, Evgeny Korsunsky and Michael Fleischhauer \\ Fachbereich Physik, Universität Kaiserslautern, D-67663 Kaiserslautern, Germany
}

(Dated: October 30, 2018)

\begin{abstract}
Resonantly enhanced four wave mixing in double- $\Lambda$ systems is limited by ac-Stark induced nonlinear phase shifts. With counter-propagating pump fields the intensity-phase coupling has minimal impact on the dynamics, but it is of critical importance for co-propagation. The nonlinear phase terms lead to an increase of the conversion length linearly proportional to the inverse seed intensity, while without nonlinear phase-mismatch the scaling is only logarithmic. We here show that the ac-Stark contributions can be eliminated while retaining the four-wave mixing contribution by choosing a suitable five level system with appropriate detunings.
\end{abstract}

PACS numbers: 42.50.Gy, 32.80.Qk, 42.50.Hz

Ever since the cancellation of resonant linear absorption and refraction via electromagnetically induced transparency (EIT) [1] was first demonstrated, quantum and nonlinear optics have successfully been exploring the consequences. Many interesting effects have been proposed and investigated [2]. One important application of EIT is optical frequency mixing close to atomic resonances where it allows making use of the resonantly enhanced nonlinear interaction without suffering from linear absorption and refraction. It has been predicted that EIT could even lead to a new regime of nonlinear optics on the level of few light quanta [3, 4, 5].

In this paper we consider one particular EIT-based scheme, namely resonantly enhanced four wave mixing in a double lambda system as shown in Fig. 1. The two fields with (complex) Rabi frequencies $\Omega_{1}$ and $\Omega_{2}$ are initially excited and form the pump fields, while the other fields with (complex) Rabi frequencies $E_{1}$ and $E_{2}$ are generated during the interaction process. $\Omega_{1}$ and $E_{1}$ are taken to be exactly on resonance while the other two are assumed to be detuned by an amount $\Delta$. A finite detuning $\Delta$, large compared to the Rabi frequencies, Doppler broadening and decay rates from the excited states, is necessary to maximize the ratio of nonlinear gain to linear absorption. Decay from the two lower levels is considered to be negligible. Because of energy conservation all fields are in four-photon resonance. It can be shown furthermore that the contributions of the resonant transitions to the linear refractive index vanish if the fields are pairwise in two-photon resonance. Phase matching will thus favor two-photon resonance and we assume that this condition is fulfilled. Resonant four-wave mixing has been analyzed both theoretically and experimentally with co-propagating as well as counter-propagating fields [6, 7, 8, 9, 10, 11, 12, 13].

Associated with the finite detuning $\Delta$ are ac-Stark shifts which lead to intensity dependent dynamical phase shifts of the fields. These phase shifts are of minor consequence in the case where the fields are counterpropagating [13]. They do have a detrimental influence, however, for co-propagation. In the following we will concentrate on the latter situation and show how to eliminate these terms leading to a considerable improvement

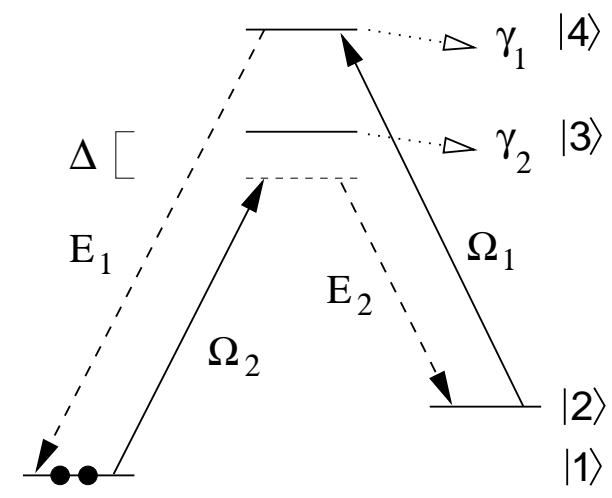

FIG. 1: Parametric amplification in a generic double- $\Lambda$ system.

of nonlinear frequency conversion.

The standard method to describe the wave mixing process in a resonant medium is to derive density matrix equations for the atomic system, solve them in the steady-state, i.e. assuming adiabaticity, and insert the resulting expressions into the Maxwell-Bloch equations. This yields four equations of motion for the fields in the slowly varying amplitude and phase approximation. The field equations can then be further broken down into a set of five coupled equations consisting of four amplitude equations plus the equation of motion governing the relative phase between the fields.

This procedure can be rather cumbersome, particularly when several atomic levels are involved. A much simpler way to derive the field equations is given by the Hamiltonian approach introduced in [14], which we will use in the following. This method makes use of the fact that the polarization $P$ of the medium can be expressed as a partial derivative of the time-averaged interaction energy per atom $H$ with respect to the electric field or, equivalently, the Rabi frequencies $E_{\mu}$

$$
P_{i}=-\frac{N d_{i}}{\hbar}\left\langle\frac{\partial H}{\partial E_{i}^{*}}\right\rangle \mathrm{e}^{-i\left(\nu_{i}(t-z / c)\right.}+c . c .
$$

A similar expression holds for the drive field polariza- 
tions with $E$ replaced by $\Omega$. Here $\langle\ldots\rangle$ denotes quantummechanical averaging, $d_{i}$ the dipole matrix elements of the corresponding transitions, and $N$ is the atomic density. Hence introducing moving coordinates $(\zeta, t)$ with $\zeta=z-c t$ one can directly obtain the field equations of motion in the slowly-varying amplitude and phase approximation:

$$
\frac{\mathrm{d} E_{i}}{\mathrm{~d} \zeta}=-i \frac{\eta_{\mu}}{\hbar}\left\langle\frac{\partial H}{\partial E_{i}^{*}}\right\rangle
$$

where $\eta_{\mu}=N d_{i}^{2} \omega_{i} /\left(2 \hbar c \epsilon_{0}\right)$. The evaluation of the right hand side of (2) is particularly simple if an open-system model can be used to incorporate decay in a complex Hamiltonian $H$ and if the atoms adiabatically follow the dynamics of the fields. If the atoms are initially in an eigenstate of $H$ with eigenvalue $\lambda$ then $\langle H\rangle$ can simply be replaced by $\lambda$ as the two are equivalent. Thus knowledge of the eigenvalues of the single-atom interaction Hamiltonian is sufficient to directly derive the field equations of motion.

In the basis $(|1\rangle|2\rangle|3\rangle|4\rangle)^{T}$ the system shown in Figure 1 1 can be described by the complex interaction Hamiltonian

$$
H=\hbar\left[\begin{array}{cccc}
0 & 0 & -\Omega_{2}^{*} & -E_{1}^{*} \\
0 & 0 & -E_{2}^{*} & -\Omega_{1}^{*} \\
-\Omega_{2} & -E_{2} & \Delta-i \gamma_{2} & 0 \\
-E_{1} & -\Omega_{1} & 0 & -i \gamma_{1}
\end{array}\right]
$$

Taking $\Omega$ as a characteristic magnitude of the Rabi frequencies involved, to second order in $\Omega / \Delta$ the relevant eigenvalue of (3) is given by

$$
\begin{array}{r}
\lambda_{0}=\frac{1}{\Delta}\left[\frac{\Omega_{1} \Omega_{2} E_{1}^{*} E_{2}^{*}+\Omega_{1}^{*} \Omega_{2}^{*} E_{1} E_{2}}{\left|\Omega_{1}\right|^{2}+\left|E_{1}\right|^{2}}\right. \\
\left.-\frac{\left|\Omega_{1}\right|^{2}\left|\Omega_{2}\right|^{2}+\left|E_{1}\right|^{2}\left|E_{2}\right|^{2}}{\left|\Omega_{1}\right|^{2}+\left|E_{1}\right|^{2}}\right] .
\end{array}
$$

This eigenvalue corresponds to the state asymptotically connected to $|1\rangle$ at $t \rightarrow-\infty$. That is, the eigenstate associated with $\lambda_{0}$ corresponds to the ground state $|1\rangle$ for vanishing $E_{1}$ and $E_{2}$. If the pump fields change sufficiently slowly we may assume that all atoms stay at all times in this eigenstate and $\langle H\rangle$ in (2) can be replaced by $\lambda_{0}$. This yields the following equations of motion 13

$$
\begin{aligned}
\frac{\partial}{\partial \zeta} E_{1}= & -i \kappa \frac{\Omega_{1}^{*} \Omega_{1}^{2} \Omega_{2} E_{2}^{*}-E_{1}^{2} E_{2} \Omega_{1}^{*} \Omega_{2}^{*}}{\Delta\left(\left|\Omega_{1}\right|^{2}+\left|E_{1}\right|^{2}\right)^{2}} \\
& -i \kappa \frac{\left|\Omega_{1}\right|^{2}\left(\left|\Omega_{2}\right|^{2}-\left|E_{2}\right|^{2}\right)}{\Delta\left(\left|\Omega_{1}\right|^{2}+\left|E_{1}\right|^{2}\right)^{2}} E_{1}, \\
\frac{\partial}{\partial \zeta} E_{2}= & -i \kappa \frac{\Omega_{1} \Omega_{2} E_{1}^{*}}{\Delta\left(\left|\Omega_{1}\right|^{2}+\left|E_{1}\right|^{2}\right)} \\
& +i \kappa \frac{\left|E_{1}\right|^{2}}{\Delta\left(\left|\Omega_{1}\right|^{2}+\left|E_{1}\right|^{2}\right)} E_{2},
\end{aligned}
$$

$$
\begin{aligned}
\frac{\partial}{\partial \zeta} \Omega_{1}= & i \kappa \frac{\Omega_{1}^{2} \Omega_{2} E_{1}^{*} E_{2}^{*}-\left|E_{1}\right|^{2} E_{1} E_{2} \Omega_{2}^{*}}{\Delta\left(\left|\Omega_{1}\right|^{2}+\left|E_{1}\right|^{2}\right)^{2}} \\
& +i \kappa \frac{\left|E_{1}\right|^{2}\left(\left|\Omega_{2}\right|^{2}+\left|E_{2}\right|^{2}\right)}{\Delta\left(\left|\Omega_{1}\right|^{2}+\left|E_{1}\right|^{2}\right)^{2}} \Omega_{1} \\
\frac{\partial}{\partial \zeta} \Omega_{2}= & -i \kappa \frac{E_{1} E_{2} \Omega_{1}^{*}}{\Delta\left(\left|\Omega_{1}\right|^{2}+\left|E_{1}\right|^{2}\right)} \\
& +i \kappa \frac{\left|\Omega_{1}\right|^{2}}{\Delta\left(\left|\Omega_{1}\right|^{2}+\left|E_{1}\right|^{2}\right)} \Omega_{2},
\end{aligned}
$$

where $\kappa=3 N \lambda^{2} \gamma / 8 \pi$, with $\gamma$ being the decay rate from the upper levels, where for simplicity we have assumed $\gamma_{1} \approx \gamma_{2}=\gamma$. Note that there are no linear absorption terms in (5) - (8), despite the presence of the decay terms $\gamma$. Thus, the process is a parametric one, and the total energy of the electromagnetic fields is conserved. One furthermore finds that the equations have the following three constants of motion:

$$
\begin{aligned}
& \left|\Omega_{1}\right|^{2}+\left|E_{1}\right|^{2}=\text { constant } \\
& \left|\Omega_{2}\right|^{2}+\left|E_{2}\right|^{2}=\text { constant } \\
& \left|\Omega_{1}\right|^{2}-\left|\Omega_{2}\right|^{2}=\text { constant. }
\end{aligned}
$$

This allows the problem to be reduced to two degrees of freedom, one corresponding to the exchange energy between the fields and the other to the relative phase $\varphi=\phi_{\Omega 1}+\phi_{\Omega 2}-\phi_{E 1}-\phi_{E 2}$ between the fields.

The terms in the second line of each equation in (5 8) are ac-Stark induced, intensity dependent phase terms. They have a considerable impact on the dynamics, particularly in terms of the conversion length, i.e. the distance required for one of the pump modes to attain maximum transfer into one of the generated modes. To see this we solve (5) - (8) analytically, using the constants of motion and the methods described in [15, 16]. We will not give details of the derivation nor the full solution here, however, as they are not very instructive for the present purposes.

We consider the case where the two pump fields are initially of equal intensity, as are the two seed fields, so that $E_{1}=E_{2}=E$ and $\Omega_{1}=\Omega_{2}=\Omega$. If we define the seed field parameter by $\epsilon=|E(0)|^{2} /|\Omega(0)|^{2}$ and denote the initial relative phase difference between the fields as $\varphi_{0}$ then, in the limit $\epsilon \ll 1$, the conversion length $L$ and conversion efficiency $e=\left(\left|E_{\max }\right|^{2}-\left|E_{\min }\right|^{2} /\left(\left|\Omega_{\max }\right|^{2}\right)\right.$ are given by:

$$
\begin{aligned}
e & =\frac{1-\cos \varphi_{0}}{1+\cos \varphi_{0}}\left[1-\epsilon \frac{1-3 \cos \varphi_{0}-2 \cos ^{2} \varphi_{0}}{1-\cos \varphi_{0}}\right] \\
L & =\frac{\Delta}{\kappa} \frac{1}{\sqrt{\epsilon}} \frac{2 \pi}{1+\cos \varphi_{0}}
\end{aligned}
$$

One immediately notices that in order to obtain full conversion as $\epsilon \rightarrow 0$ it is necessary to carefully choose the initial phase $\varphi_{0}$. Secondly the conversion length scales as $L \sim 1 / \sqrt{\epsilon}$, that is, inversely in the seed field amplitude $E(0)$. Consequently for small, let alone vacuum, seed fields, the conversion distance will rapidly become infeasibly long. 
On the other hand, if the phase terms in $(5-8)$ were to be omitted, the quantity

$$
\operatorname{Re}\left(\Omega_{1} \Omega_{2} E_{1}^{*} E_{2}^{*}\right)=\left|\Omega_{1} \Omega_{2} E_{1}^{*} E_{2}^{*}\right| \cos \varphi,
$$

is another constant of motion. In this case, if one of the generated fields $E_{1}, E_{2}$ vanishes initially, i.e. if only one of these fields is seeded, then this constant of motion must be zero. This indicates that the relative phase $\varphi$ can only jump discontinuously between $\pm \pi / 2$, which occurs only at the end of each conversion cycle, when at least one of the field amplitudes vanishes. Thus in this case the phase is essentially decoupled from the evolution of the field amplitudes. This makes a considerable difference to the dynamics. From an analytic solution one finds for the conversion efficiency $e$ and the conversion length $L$

$$
\begin{aligned}
e & =1-\epsilon \sqrt{\cos \varphi_{0}}, \\
L & =\frac{2 \Delta}{\kappa} \log \left(\frac{4}{\epsilon^{2} \cos \varphi_{0}}\right)
\end{aligned}
$$

where we have taken $0<\cos \varphi_{0}<1$ for simplicity. Thus if the phase terms are not present, it is possible to obtain full conversion in the small seed field limit, regardless of the initial phase condition. Secondly, the conversion distance scales only as $-\log \epsilon$, and the situation is completely different to the previous case - the conversion length will always remain short.

As an illustration of conversion distance dependence on seed field intensity we have calculated numerical solutions to Eqs. (5) - (8) with and without the phase terms, and without making the approximation that $\epsilon \ll 1$. We have assumed $E_{1}=E_{2}, \Omega_{1}=\Omega_{2}$ and $\varphi_{0}=\pi / 4$. The results are shown in Figure 2.



FIG. 2: Initial field intensities taken as $\left|\Omega_{1}(0)\right|^{2}=\left|\Omega_{2}(0)\right|^{2}$, $\left|E_{1}(0)\right|^{2}=\left|E_{2}(0)\right|^{2}$, with the seed field intensity defined as $\epsilon=\left|E_{1}\right|^{2} /\left|\Omega_{1}^{2}\right|$.

Thus, given the obvious advantages inherent in the omission of these phase terms, the question naturally arises: does there exist a situation in which these terms can be made to vanish?
It has been shown by Harris 177 that in a system of parallel $\lambda$ transitions with different excited-state energies there exists an optimum detuning such that the nonlinear index of refraction vanishes. A similar idea can be applied here. Noting that both parts of (田) are linear in $\Delta$, but only the first part is linear in each of the fields, suggests a method for canceling the phase terms and at the same time retaining the nonlinear interaction part. To see this, consider the five level system shown in Figure 3. Here we have introduced hyperfine angu-

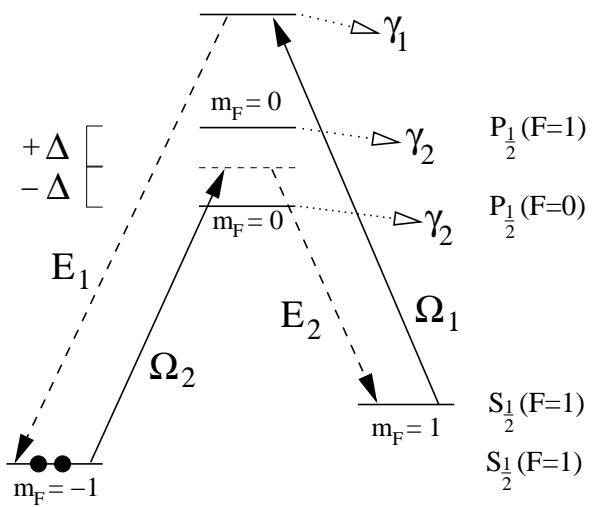

FIG. 3: Modified double- $\Lambda$ system. $F$ and $m_{F}$ represent the total and $z$-projection angular momentum for the atom, including hyper-fine interaction.

lar momentum terms, level $|3\rangle$ in the original scheme has been split into two and the pump beam $\Omega_{2}$ tuned to a point midway between them. Within the approximation that the electronic parts of the wave functions describing the two $P_{1 / 2}$ states are identical, as are the electronic parts of the two $S_{1 / 2}$ states, we find that the coupling strengths for the $\left|S_{1 / 2}\right\rangle \rightarrow\left|P_{1 / 2}\right\rangle$ transitions all have the same magnitude. Crucially, however, the $\left|S_{1 / 2}, F=1, m_{F}=-1\right\rangle \rightarrow\left|P_{1 / 2}, F=1, m_{F}=0\right\rangle$ transition has opposite sign to the other three. The Hamiltonian thus becomes

$$
H=-\hbar\left[\begin{array}{ccccc}
0 & 0 & \Omega_{2}^{*} & \Omega_{2}^{*} & E_{1}^{*} \\
0 & 0 & E_{2}^{*} & -E_{2}^{*} & \Omega_{1}^{*} \\
\Omega_{2} & E_{2} & -\Delta-i \gamma_{2} & 0 & 0 \\
\Omega_{2} & -E_{2} & 0 & \Delta-i \gamma_{2} & 0 \\
E_{1} & \Omega_{1} & 0 & 0 & -i \gamma_{1}
\end{array}\right]
$$

We now find that to second order in $\Omega / \Delta$ the lowest eigenvalue of (17) is

$$
\lambda_{0}=\frac{1}{\Delta}\left[\frac{\Omega_{1} \Omega_{2} E_{1}^{*} E_{2}^{*}+\Omega_{1}^{*} \Omega_{2}^{*} E_{1} E_{2}}{\left|\Omega_{1}\right|^{2}+\left|E_{1}\right|^{2}}\right] .
$$

We see that the phase terms responsible for the increase in the conversion length and sensitive dependence of the conversion efficiency on the initial phase are indeed absent. It should be noted that under more general conditions, e.g. if the dipole moments to the $F=1$ and $F=0$ manifolds are not equal, other values of the detunings need to be chosen. 
In summary we have shown that the nonlinear phase contributions arising in resonant forward four-wave mixing due to the ac-Stark effect can be exactly eliminated if a five state system with appropriate couplings and detunings is used. We have derived a simple effective Hamilto- nian for this system and shown that the conversion length scales only logarithmically with the inverse seed intensity whereas with the phase terms present conversion length scales linearly.
[1] S. E. Harris, Physics Today 50, 36 (1997).

[2] E. Arimondo, Prog. in Optics, 35, 259 (1996), P. L. Knight, B. Stoicheff and D. F. Walls (eds.), Phil. Trans. R. Soc. Lond. A 355, 2215-2416 (1997), J. P. Marangos, Journal of Modern Optics, 45, 471 (1998),

[3] S. E. Harris and Y. Yamamoto, Phys. Rev. Lett. 81, 3611 (1998)

[4] S. E. Harris and L. V. Hau, Phys. Rev. Lett. 82, 4611 (1999)

[5] H. Schmidt and A. Imamoglu, Opt. Lett. 21, 1936 (1996), M. D. Lukin and A. Imamoglu, Phys. Rev. Lett. 84, 1419 (2000).

[6] P.R. Hemmer, K. Z. Cheng, J. Kierstead, M. S. Shariar, and M. K. Kim, Opt. Lett. 19, 296 (1994); B. S. Ham, M. S. Shariar, M. K. Kim, and P. R. Hemmer, Opt. Lett. 22, 1849 (1997).

[7] S. Babin, U. Hinze, E. Tiemann, B. Wellegehausen, Opt. Lett. 21, 1186 (1996); S. Babin, E. V. Podivilov, D.A. Shapiro, U. Hinze, E. Tiemann, B. Wellegehausen, Phys. Rev. A 59, 1355 (1999); U. Hinze, L. Meyer, B.N. Chichkov, E. Tiemann, B. Wellegehausen, Opt. Comm 166, 127 (1999).

[8] M. D. Lukin, M. Fleischhauer, A.S. Zibrov, H.G. Robinson, V.L. Velichansky, L. Hollberg, M.O. Scully, Phys.
Rev. Lett. 79, 2959 (1997).

[9] A. K. Popov, S. A. Myslivets, Kvant. Elektr. 24, 1033 (1997).

[10] B. Lu, W.H. Burkett, M. Xiao, Opt. Lett. 23, 804 (1998);

[11] M. D. Lukin, P. R. Hemmer, M. Löffler and M. O. Scully Phys. Rev. Lett. 81, 2675 (1998); A. S. Zibrov, M. D. Lukin and M. O. Scully Phys. Rev. Lett. 83, 4049 (1999).

[12] E. A. Korsunsky and D. V. Kosachiov, Phys. Rev. A 60, 4996 (1999).

[13] M. Fleischhauer, in Frontiers of Laser Physics and Quantum Optics, ed. by Z. Xu, S. Xie, S.-Y. Zhu and M.O. Scully, pp. 97 - 106 (Springer, Berlin, 2000).

[14] A.O. Melikyan and S.G. Saakyan, Zh. Exp.Teor. Fiz. 76, 1530 (1979) [Sov. Phys. JETP 49, 776 (1979)]; A.R. Karapetyan and B.V. Kryzhanovskii, Zh. Exp. Teor. Fiz. 99, 1103 (1991) [Sov. Phys. JETP 72, 613 (1991)]; B. Kryzhanovsky and B. Glushko, Phys. Rev. A 45, 4979 (1992).

[15] E. A. Korsunsky and M. F. Fleischhauer, quant$\mathrm{ph} / 0204089$.

[16] B. Kryzhanovsky and B. Glushko, Phys. Rev. A 45, 4979 (1992).

[17] S. E. Harris, Opt. Lett. 19, 2018 (1994). 